\title{
EOPs: anticonceptivos orales y eventos tromboembólicos
}

\section{Caso Clínico}

Un Residente de segundo año atiende en la guardia a una paciente de 25 años previamente sana con un cuadro compatible con trombosis venosa axilar. Sorprendido por el hallazgo en una mujer joven sin antecedentes, consulta con el Jefe de Residentes quien le sugiere averiguar si la paciente recibía anticonceptivos orales. Al interrogarla, la mujer refiere que recibía anticonceptivos con baja dosis de etinilestradiol desde hacía tres años. Como el Residente no sabía cómo modificaba la probabilidad previa de trombosis este nuevo dato del interrogatorio, decidió realizar una búsqueda bibliográfica.

\section{Preguntas que generó la paciente}

El uso de anticonceptivos orales (exposición) en mujeres jóvenes (la población), ¿se asocia con mayor riesgo de desarrollar un fenómeno tromboembólico? (el resultado).

\section{Búsqueda bibliográfica}

Se realizó una búsqueda en Pub Med utilizando filtros metodológicos (clinical queries), acotándose la misma a estudios de cohortes y caso-control que permitiran establecer asociación entre la exposición y el resultado.

Los terminos MESH empleados fueron: "contraceptives oral"; "trombophlebitis venous"; "women", seleccionándose dos trabajos, uno de ellos un metaanálisis.

\section{Anticonceptivos orales y trombosis venosa profunda}

La Trombosis Venosa Profunda (TVP) es una patología vascular común con una incidencia en EEUU de 800.000 casos/ año. Se produce por la oclusión de las venas producto de un agregado plaquetario sumado a depósitos de fibrina y elementos formes de la sangre. La causa no está del todo aclarada, aunque en la mayor parte de los casos, obedece a la presencia de algunos de los elementos de la tríada de Virchow (estasis, hipercoagulabilidad, daño intimal). El diagnóstico clínico es dificil y poco fiable presentando signos y síntomas en sólo el $50 \%$ de los casos.

Los Anticonceptivos Orales (ACO) son utilizados por más de 200 millones de mujeres en todo el mundo y por 1 de cada 4 mujeres menores de 45 años en EE.UU. La popularidad de las píldoras se debe a la facilidad en la administración, a la reducida tasa de de fracaso del método $(<1 \%$ ) y a la baja frecuencia de efectos adversos. Actúan inhibiendo la ovulación por supresión de la secreción de LH y FSH; alteran el moco cervical; reducen la movilidad y las secreciones del oviducto y del útero.

Se sabe que la tasa de mortalidad en mujeres menores de 40 años que utilizan ACO es inferior a la de las mujeres que no utilizan ningún método anticonceptivo. Esta diferencia es atribuida al aumento de la mortalidad asosiada al embarazo. Esta disminución es aún más notable en las mujeres no fumadoras respecto de las fumadoras que toman ACO.

La tasa de mortalidad de las mujeres del grupo etario comprendido entre 15-25 años no tabaquista que utiliza ACO es inferior a la de mujeres que utilizan otros métodos de control de la fertilidad, obedeciendo esto a la mayor tasa de fracaso y al riesgo consecuente de embarazo.

A pesar de la inocuidad global, las usuarias de ACO muestran cierto riesgo de presentar algunos efectos colaterales.

La mayor parte de los estudios prospectivos y retrospectivos han demostrado mayor incidencia de enfermedad tromboembólica (TVP-TEP). El Riesgo Relativo (RR) en diversas publicaciones varía entre 2 y 5 (mayor riesgo en las usadoras de ACO) siendo mayor en mujeres que ingieren los ACO antiguos con $50 \mathrm{mcg}$ de estrógenos.
A pesar de este elevado riesgo relativo, el impacto poblacional es pequeño debido a que estamos ante un evento muy infrecuente (TVP-TEP en población joven).

La explicación fisipatológica de este fenómeno se relaciona con el hecho de que los ACO (el componente estrogénico) favorecen los factores procoagulantes evidenciándose una disminución de los valores séricos de antitrombina III.

A su vez, el hábito tabáquico y la edad aumentan la morbimortalidad por las complicaciones de la enfermedad tromboembólica, de ahí la usual cautela en el uso de ACO en fumadoras mayores de 35/40 años.

\section{Resumen de los trabajos seleccionados}

1. Farmer R, Lawrenson R, Thompson D, Kenedy J: Population-based study of risk of venous thromboembolism associated with various oral contraceptives. Lancet an. 11,1997, vol.349: Este estudio poblacional se basó en los registros médicos de 540.000 mujeres del Reino Unido, nacidas entre 1941-1981. Las mujeres enroladas tuvieron el diagnóstico de: TVP, trombosis no especificada, TEP, evento trombótico potencial en tratamiento empírico con anticoagulantes.

Los criterios de exclusión fueron: TVP previa, historia de trauma, cirugía reciente (90 días), embarazo y puerperio.

Este estudio se dividió en dos partes: a) estudio de cohortes donde se analizó y comparó la incidencia de fenómenos venosos trombóticos en mujeres usuarias de los AC0 comunes. b) estudio casocontrol donde fue calculado el odds ratio de TVP y la asociación con diferentes tipos de ACO ajustando por posibles variables confundidoras.

La incidencia cruda de eventos venosos tromboembólicos por cada 10.000 mujeres-año fue de 4.10 en usuarias corrientes de algún AC0; 3.10 en usuarias de ACO de segunda generación y 4.98 en las usuarias de ACO de tercera generación. Las diferencias entre ACO de segunda y tercera generación no fueron significativas.

2. Multicenter study: Venous thromboembolic disease and combined oral contraceptives result of international multicenter casecontrol study. World health organization colaborative study of cardiovascular disease and steroid hormone contraception. Lancet December 16,1995, vol 346:

Este reporte describe el riesgo de eventos tromboembólicos venosos idiopáticos (TVP, TEP, etc) en asociación con el uso corriente de ACO. Fueron evaluados 1143 casos de entre 20-44 años de edad y 2998 controles en el estudio caso-control en 21 centros de Africa, Asia, Europa y Latinoamérica. Los casos fueron categorizados según el grado de certeza en TVP definida, TVP probable, TVP posible, y TEP definido, probable, o posible.

El análisis reveló un aumento de riesgo de tromboembolismo venoso en mujeres europeas (odds ratio 4.14; IC 95\% 3.09-5.57) y en mujeres del resto de los países (odds ratio 3.25; IC 95\% 2.594.08).

El estudio confirmó la asociación entre el uso de ACO y los eventos trombóticos venosos. Además mostró aumento del riesgo en las mujeres tabaquistas ( $>10$ cigarrillos por dia) y en las mujeres con indice se masa corporal $>25$.

\section{Comentario final}

Ambos estudios mostraron aumento en la incidencia de eventos tromboembólicos venosos en la población de mujeres que utilizaban ACO, si bien los dos trabajos seleccionados coinciden en que no se observan las diferencias comunicadas en los estudios pre- 
vios. Esta diferencia puede explicarse por la presencia de sesgos metodológicos.

Con respecto a los anticonceptivos orales de tercera generación, cuyo componente progestacional es el Desorgestrel y Gestodeno, se ha encontrado un aumento en la incidencia de eventos trombóticos comparados con los ACO que tienen como complemento progestacional el levonorgestrel. Al realizar el análisis multivaria$b e^{*}$, tomando en cuenta las posibles variables confundidoras, no se encontraron diferencias significativas entre uno y otro. El aumento observado inicialmente pudo deberse a la selección en el riesgo cardiovascular de mujeres ya que es probable que aquéllas con mas riesgo hubieran recibido ACO de tercera generación por existir cierta evidencia de que estos ACO son mas seguros por conferir protección para eventos cardiovasculares.

De todos modos, a pesar de que este aumento en el riesgo es estadísticamente significativo, su implicancia clínica no parece demasiado importante. Este pequeño aumento (en términos absolutos) de episodios tromboembólicos no cambiará en general la decisión de recomendar los ACO, ya que debemos tomar en cuenta la mayor morbimortalidad en las mujeres en edad fértil por patologías asociadas con el embarazo. También es importante notar el riesgo de TVP asociado a la gestación, que es en definitiva el evento que se quiere evitar con la toma de los ACO. Mientras en el embarazo la incidencia es de 60 por 100.000/año, en la población general y en las usuarias de ACO es de 5 por 100.000/año y 15 por 100.000/año respectivamente.

La utilidad podría residir en seleccionar el grupo de pacientes donde el uso de ACO estaría asociado a mayores complicaciones (fumadoras, mayores) y por ende a mayor morbimortalidad. En ellas se puede recomendar la discontinuacion de los ACO ante situaciones que incrementen el riesgo de los eventos tromboembolicos como cirugias pelvianas, fracturas asociadas a inmovilidad, injurias severas, inmovilización, etc, donde se adicionarían condiciones procoagulantes.

*Ver glosario

Dr. Matias Deprati

Residente. Unidad de Medicina Familiar y Preventiva. Hospital Italiano de Buenos Aires.

\section{Concurso de Fotografía}

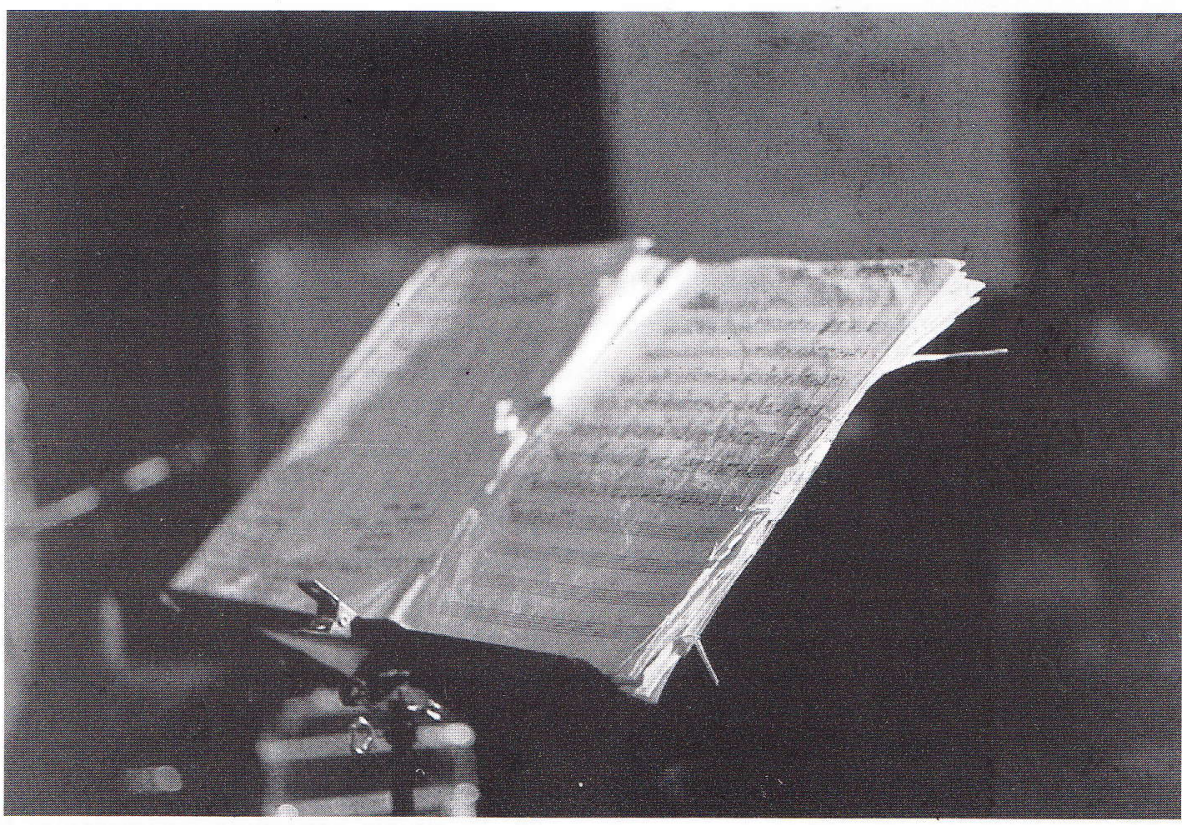

Dra. Micaela Gago

Médica de Familia. Buenos Aires. Argentina 